\title{
Graduation Rates and Accountability: Regressions Versus Production Frontiers
}

\author{
Robert B. Archibald · David H. Feldman
}

Published online: 13 October 2007

(C) Springer Science+Business Media, LLC 2007

\section{Erratum to: Res High Educ (2007) DOI 10.1007/s11162-007-9063-6}

The table of results in Appendix 2 that appeared in the original online and print versions of this paper contains an error. The final four columns of Appendix 2 report the input slacks. Our frontier model was output-oriented and both the technical efficiency scores and input slacks were calculated in that framework. Yet the slacks that we put in the table came from an input-oriented calculation. This was simply a clerical error on our part in the production of the final table. When we referred to the slacks in the paper, we were using the correct numbers that appear in this erratum. Thus the error in the printed table has no effect on our conclusions or our methodology.

A casual perusal of the two sets of slacks shows that they are not the same, and in a small numbers of cases the differences are substantial. An output-oriented efficiency measure reports how much output could be increased without altering input quantities used by projecting an inefficient firm to the frontier. An input-oriented efficiency measure reports how much inputs could be proportionately reduced without affecting output. Using Fig. 2, output-oriented DEA projects up from inefficient firms 4, 5, 6, and 7 vertically to the frontier. Thus there is a significant amount of input slack for firm 7. On the other hand, input-oriented DEA would project horizontally leftward back to the frontier. In this case there is no input slack for any of the four inefficient examples. This accounts for why slack measures can differ and reinforces why context is important in interpreting the input slack.

The online version of the original article can be found under doi: 10.1007/s11162-007-9063-6.

R. B. Archibald $(\bowtie) \cdot$ D. H. Feldman

Department of Economics, College of William and Mary, Williamsburg, VA 23187-8795, USA

e-mail: rbarch@wm.edu

D. H. Feldman

e-mail: dhfeld@wm.edu 


\section{Appendix}

Appendix 2 Table of results

\begin{tabular}{|c|c|c|c|c|c|c|c|c|}
\hline \multicolumn{2}{|l|}{ Rank } & \multirow[t]{2}{*}{ School } & \multirow[t]{2}{*}{ Resid. } & \multirow[t]{2}{*}{ TE } & \multicolumn{4}{|c|}{ Input slacks } \\
\hline DEA & RES & & & & SAT & Top10 & Full & Cost \\
\hline 1 & 8 & Auburn Univ. & 0.555 & 1 & 0 & 0 & 0 & 0 \\
\hline 1 & 13 & Bowling Green State Univ. & 0.485 & 1 & 0 & 0 & 0 & 0 \\
\hline 1 & 5 & Brown Univ. & 0.830 & 1 & 0 & 0 & 0 & 0 \\
\hline 1 & 51 & Florida State Univ. & 0.195 & 1 & 0 & 0 & 0 & 0 \\
\hline 1 & 4 & Fordham Univ. & 0.840 & 1 & 0 & 0 & 0 & 0 \\
\hline 1 & 1 & Harvard Univ. & 1.374 & 1 & 0 & 0 & 0 & 0 \\
\hline 1 & 10 & Howard Univ. & 0.500 & 1 & 0 & 0 & 0 & 0 \\
\hline 1 & 34 & Illinois State Univ. & 0.268 & 1 & 0 & 0 & 0 & 0 \\
\hline 1 & 84 & Indiana State Univ. & 0.049 & 1 & 0 & 0 & 0 & 0 \\
\hline 1 & 20 & Indiana Univ.-Bloomington & 0.390 & 1 & 0 & 0 & 0 & 0 \\
\hline 1 & 68 & Johns Hopkins Univ. & 0.109 & 1 & 0 & 0 & 0 & 0 \\
\hline 1 & 107 & Louisiana State Univ._-Baton Rouge & -0.053 & 1 & 0 & 0 & 0 & 0 \\
\hline 1 & 56 & Louisiana Tech Univ. & 0.170 & 1 & 0 & 0 & 0 & 0 \\
\hline 1 & 72 & Mississippi State Univ. & 0.090 & 1 & 0 & 0 & 0 & 0 \\
\hline 1 & 73 & New School Univ. & 0.086 & 1 & 0 & 0 & 0 & 0 \\
\hline 1 & 121 & Oklahoma State Univ. & -0.133 & 1 & 0 & 0 & 0 & 0 \\
\hline 1 & 15 & Pace Univ. & 0.433 & 1 & 0 & 0 & 0 & 0 \\
\hline 1 & 19 & Pennsylvania State Univ. & 0.396 & 1 & 0 & 0 & 0 & 0 \\
\hline 1 & 2 & St. John's Univ. & 0.879 & 1 & 0 & 0 & 0 & 0 \\
\hline 1 & 33 & SUNY-Albany & 0.274 & 1 & 0 & 0 & 0 & 0 \\
\hline 1 & 16 & Syracuse Univ. & 0.431 & 1 & 0 & 0 & 0 & 0 \\
\hline 1 & 118 & Texas A\&M Univ.-Commerce & -0.112 & 1 & 0 & 0 & 0 & 0 \\
\hline 1 & 76 & Univ. of Akron & 0.074 & 1 & 0 & 0 & 0 & 0 \\
\hline 1 & 35 & Univ. of California-Davis & 0.266 & 1 & 0 & 0 & 0 & 0 \\
\hline 1 & 54 & Univ. of California-Irvine & 0.181 & 1 & 0 & 0 & 0 & 0 \\
\hline 1 & 57 & Univ. of Colorado-Denver & 0.167 & 1 & 0 & 0 & 0 & 0 \\
\hline 1 & 113 & Univ. of Georgia & -0.083 & 1 & 0 & 0 & 0 & 0 \\
\hline 1 & 40 & Univ. of Illinois-Urbana-Champagne & 0.243 & 1 & 0 & 0 & 0 & 0 \\
\hline 1 & 131 & Univ. of Louisville & -0.165 & 1 & 0 & 0 & 0 & 0 \\
\hline 1 & 95 & Univ. of Missouri-St. Louis & -0.013 & 1 & 0 & 0 & 0 & 0 \\
\hline 1 & 14 & Univ. of New Hampshire & 0.433 & 1 & 0 & 0 & 0 & 0 \\
\hline 1 & 6 & Univ. of Notre Dame & 0.825 & 1 & 0 & 0 & 0 & 0 \\
\hline 1 & 55 & Univ. of Vermont & 0.172 & 1 & 0 & 0 & 0 & 0 \\
\hline 1 & 3 & Univ. of Virginia & 0.843 & 1 & 0 & 0 & 0 & 0 \\
\hline 1 & 101 & Univ. of Wisconsin-Milwaukee & -0.032 & 1 & 0 & 0 & 0 & 0 \\
\hline 36 & 27 & Clemson Univ. & 0.330 & 0.999 & 11 & 0 & 0 & 0 \\
\hline 36 & 58 & Univ. of California-Riverside & 0.148 & 0.999 & 0 & 0.459 & 0.066 & 0 \\
\hline 36 & 87 & Univ. of Northern Colorado & 0.029 & 0.999 & 0 & 0 & 0.104 & 0 \\
\hline 39 & 24 & Univ. of Wisconsin-Madison & 0.366 & 0.995 & 0 & 0 & 0.040 & 0 \\
\hline
\end{tabular}


Appendix 2 continued

\begin{tabular}{|c|c|c|c|c|c|c|c|c|}
\hline \multicolumn{2}{|l|}{ Rank } & \multirow[t]{2}{*}{ School } & \multirow[t]{2}{*}{ Resid. } & \multirow[t]{2}{*}{$\mathrm{TE}$} & \multicolumn{4}{|c|}{ Input slacks } \\
\hline DEA & RES & & & & SAT & Top10 & Full & Cost \\
\hline 39 & 53 & Miami Univ.—Oxford & 0.182 & 0.995 & 41 & 0 & 0.136 & 0 \\
\hline 41 & 39 & Tufts Univ. & 0.243 & 0.994 & 24 & 0 & 0.061 & 0 \\
\hline 42 & 9 & Georgetown Univ. & 0.520 & 0.992 & 0 & 0 & 0.021 & 0 \\
\hline 42 & 52 & Ohio Univ. & 0.186 & 0.992 & 10 & 0 & 0.032 & 0 \\
\hline 44 & 32 & Lehigh Univ. & 0.282 & 0.991 & 0 & 0 & 0 & 1194 \\
\hline 45 & 11 & College of William and Mary & 0.494 & 0.989 & 6 & 0 & 0.043 & 0 \\
\hline 46 & 21 & Yale Univ. & 0.387 & 0.987 & 0 & 0.057 & 0.074 & 17515 \\
\hline 47 & 12 & Northwestern Univ. & 0.489 & 0.985 & 0 & 0.026 & 0 & 2945 \\
\hline 48 & 17 & Pepperdine Univ. & 0.412 & 0.984 & 0 & 0.183 & 0 & 4730 \\
\hline 49 & 31 & Univ. of Michigan-Ann Arbor & 0.291 & 0.981 & 0 & 0 & 0.086 & 0 \\
\hline 50 & 25 & Seton Hall Univ. & 0.356 & 0.980 & 0 & 0 & 0 & 2687 \\
\hline 50 & 46 & Virginia Tech & 0.222 & 0.980 & 0 & 0 & 0.045 & 0 \\
\hline 52 & 41 & Dartmouth Univ. & 0.239 & 0.979 & 0 & 0 & 0.078 & 14571 \\
\hline 53 & 7 & Univ. of Missouri-Kansas City & 0.578 & 0.976 & 0 & 0.008 & 0 & 710 \\
\hline 53 & 30 & Univ. of Delaware & 0.313 & 0.976 & 0 & 0 & 0.079 & 2130 \\
\hline 55 & 23 & Stanford Univ. & 0.371 & 0.975 & 0 & 0.009 & 0 & 2113 \\
\hline 56 & 22 & Michigan State Univ. & 0.376 & 0.973 & 0 & 0 & 0 & 0 \\
\hline 56 & 43 & DePaul Univ. & 0.227 & 0.973 & 17 & 0 & 0 & 0 \\
\hline 58 & 29 & Duquesne Univ. & 0.319 & 0.972 & 0 & 0.123 & 0 & 0 \\
\hline 59 & 82 & Colorado State Univ. & 0.057 & 0.970 & 66 & 0 & 0 & 0 \\
\hline 60 & 28 & Columbia Univ. & 0.320 & 0.969 & 0 & 0.027 & 0.003 & 0 \\
\hline 61 & 143 & Wake Forest Univ. & -0.237 & 0.968 & 0 & 0 & 0.057 & 15509 \\
\hline 62 & 70 & Univ. of California—Los Angeles & 0.098 & 0.967 & 0 & 0.131 & 0.013 & 0 \\
\hline 63 & 59 & Univ. of Pennsylvania & 0.134 & 0.964 & 0 & 0.048 & 0 & 7321 \\
\hline 64 & 26 & Univ. of Connecticut & 0.342 & 0.963 & 0 & 0 & 0.021 & 0 \\
\hline 65 & 36 & Marquette Univ. & 0.257 & 0.961 & 5 & 0 & 0.123 & 0 \\
\hline 65 & 65 & Duke Univ. & 0.121 & 0.961 & 0 & 0.034 & 0.048 & 0 \\
\hline 67 & 127 & Univ. of Houston & -0.152 & 0.960 & 0 & 0.042 & 0 & 0 \\
\hline 68 & 71 & Univ. of North Carolina_-Chapel Hill & 0.097 & 0.958 & 0 & 0 & 0.016 & 0 \\
\hline 68 & 96 & Univ. of California_-Santa Barbara & -0.016 & 0.958 & 0 & 0.234 & 0.034 & 0 \\
\hline 70 & 120 & Washington Univ. in St. Louis & -0.130 & 0.954 & 0 & 0 & 0 & 16540 \\
\hline 71 & 18 & Univ. of Denver & 0.410 & 0.952 & 0 & 0.004 & 0 & 0 \\
\hline 71 & 135 & Univ. of Chicago & -0.170 & 0.952 & 0 & 0 & 0.022 & 4288 \\
\hline 73 & 145 & Massachusetts Inst. of Technology & -0.245 & 0.948 & 30 & 0.067 & 0.099 & 0 \\
\hline 74 & 60 & SUNY_Binghamton & 0.134 & 0.945 & 0 & 0 & 0.106 & 0 \\
\hline 75 & 112 & Univ. of California-San Diego & -0.081 & 0.944 & 0 & 0.117 & 0.018 & 0 \\
\hline 76 & 42 & West Virginia Univ. & 0.232 & 0.942 & 0 & 0.031 & 0.012 & 0 \\
\hline 76 & 115 & Rensselaer Polytechnic Institute & -0.098 & 0.942 & 6 & 0 & 0.059 & 0 \\
\hline 78 & 45 & Univ. of South Carolina—Columbia & 0.223 & 0.940 & 0 & 0.065 & 0 & 0 \\
\hline 78 & 119 & Univ. of Central Florida & -0.113 & 0.940 & 61 & 0 & 0 & 0 \\
\hline 80 & 63 & Univ. of the Pacific & 0.127 & 0.935 & 0 & 0 & 0.086 & 0 \\
\hline 80 & 77 & Texas A\&M Univ.-College Station & 0.069 & 0.935 & 0 & 0 & 0.104 & 0 \\
\hline
\end{tabular}


Appendix 2 continued

\begin{tabular}{|c|c|c|c|c|c|c|c|c|}
\hline \multicolumn{2}{|l|}{ Rank } & \multirow[t]{2}{*}{ School } & \multirow[t]{2}{*}{ Resid. } & \multirow[t]{2}{*}{$\mathrm{TE}$} & \multicolumn{4}{|c|}{ Input slacks } \\
\hline DEA & RES & & & & SAT & Top10 & Full & Cost \\
\hline 82 & 141 & Rice Univ. & -0.232 & 0.934 & 0 & 0.006 & 0.025 & 0 \\
\hline 82 & 147 & Brandeis Univ. & -0.265 & 0.934 & 0 & 0 & 0.067 & 0 \\
\hline 82 & 153 & Vanderbilt Univ. & -0.284 & 0.934 & 32 & 0 & 0.070 & 0 \\
\hline 85 & 90 & Univ. of Southern California & 0.018 & 0.933 & 0 & 0 & 0.034 & 0 \\
\hline 86 & 105 & North Dakota State Univ. & -0.047 & 0.931 & 0 & 0 & 0.103 & 0 \\
\hline 87 & 74 & Univ. of Massachusetts_-Amherst & 0.083 & 0.929 & 0 & 0 & 0.005 & 0 \\
\hline 88 & 75 & Univ. of San Francisco & 0.083 & 0.925 & 3 & 0 & 0 & 0 \\
\hline 89 & 104 & Univ. of New Mexico & -0.041 & 0.924 & 0 & 0.004 & 0 & 0 \\
\hline 90 & 149 & Emory Univ. & -0.271 & 0.923 & 0 & 0.032 & 0 & 5466 \\
\hline 91 & 50 & Univ. of South Dakota & 0.198 & 0.917 & 0 & 0 & 0 & 0 \\
\hline 92 & 47 & Purdue Univ._-West Lafayette & 0.205 & 0.916 & 0 & 0 & 0.094 & 0 \\
\hline 93 & 126 & Univ. of California-Berkeley & -0.148 & 0.915 & 0 & 0.144 & 0.035 & 0 \\
\hline 94 & 81 & Univ. of Iowa & 0.059 & 0.914 & 11 & 0 & 0 & 0 \\
\hline 94 & 99 & Univ. of Florida & -0.030 & 0.914 & 0 & 0 & 0.068 & 0 \\
\hline 96 & 78 & Univ. of Washington & 0.062 & 0.913 & 0 & 0 & 0.142 & 0 \\
\hline 97 & 80 & George Washington Univ. & 0.059 & 0.909 & 16 & 0 & 0 & 0 \\
\hline 98 & 151 & Worcester Polytechnic Institute & -0.282 & 0.908 & 9 & 0 & 0.024 & 0 \\
\hline 98 & 186 & California Institute of Technology & -0.905 & 0.908 & 20 & 0.100 & 0.114 & 17759 \\
\hline 100 & 83 & Univ. of Oregon & 0.054 & 0.905 & 0 & 0 & 0.032 & 0 \\
\hline 101 & 44 & Western Michigan Univ. & 0.223 & 0.902 & 0 & 0 & 0 & 0 \\
\hline 101 & 86 & Iowa State Univ. & 0.039 & 0.902 & 0 & 0 & 0.032 & 0 \\
\hline 103 & 69 & Northern Illinois Univ. & 0.105 & 0.899 & 0 & 0 & 0 & 0 \\
\hline 103 & 88 & American Univ. & 0.025 & 0.899 & 30 & 0 & 0.109 & 1369 \\
\hline 105 & 116 & Univ. of Texas-Austin & -0.108 & 0.898 & 0 & 0 & 0 & 0 \\
\hline 106 & 48 & Univ. of Alabama & 0.203 & 0.896 & 0 & 0 & 0.010 & 0 \\
\hline 107 & 166 & New York Univ. & -0.388 & 0.893 & 17 & 0 & 0 & 0 \\
\hline 108 & 64 & Loyola Univ. Chicago & 0.121 & 0.889 & 0 & 0 & 0.023 & 6154 \\
\hline 108 & 109 & Kent State Univ. & -0.062 & 0.889 & 0 & 0 & 0.007 & 0 \\
\hline 110 & 181 & Univ. of Rochester & -0.568 & 0.888 & 1 & 0 & 0.045 & 2652 \\
\hline 111 & 38 & St. Louis Univ. & 0.245 & 0.887 & 3 & 0 & 0 & 0 \\
\hline 112 & 117 & Univ. of San Diego & -0.109 & 0.886 & 0 & 0 & 0.105 & 0 \\
\hline 113 & 183 & Carnegie Mellon Univ. & -0.654 & 0.884 & 0 & 0 & 0.056 & 0 \\
\hline 114 & 124 & Baylor Univ. & -0.144 & 0.882 & 0 & 0 & 0.191 & 0 \\
\hline 115 & 100 & Clark Univ. & -0.031 & 0.881 & 0 & 0 & 0.116 & 2492 \\
\hline 115 & 144 & Univ. of Montana & -0.238 & 0.881 & 0 & 0 & 0.129 & 0 \\
\hline 117 & 138 & Univ. of Texas-Dallas & -0.203 & 0.880 & 39 & 0 & 0 & 0 \\
\hline 118 & 123 & Univ. of Maryland-College Park & -0.144 & 0.878 & 0 & 0 & 0.020 & 0 \\
\hline 119 & 62 & Univ. of Maine-Orono & 0.127 & 0.876 & 0 & 0 & 0 & 0 \\
\hline 119 & 171 & Tulane Univ. & -0.428 & 0.876 & 43 & 0 & 0.021 & 0 \\
\hline 121 & 125 & Hofstra Univ. & -0.144 & 0.873 & 0 & 0 & 0 & 3328 \\
\hline 122 & 66 & Univ. of Toledo & 0.112 & 0.872 & 0 & 0 & 0 & 0 \\
\hline 123 & 91 & Univ. of Nebraska-Lincoln & 0.016 & 0.871 & 22 & 0 & 0 & 0 \\
\hline
\end{tabular}


Appendix 2 continued

\begin{tabular}{|c|c|c|c|c|c|c|c|c|}
\hline \multicolumn{2}{|l|}{ Rank } & \multirow[t]{2}{*}{ School } & \multirow[t]{2}{*}{ Resid. } & \multirow[t]{2}{*}{ TE } & \multicolumn{4}{|c|}{ Input slacks } \\
\hline DEA & RES & & & & SAT & Top10 & Full & Cost \\
\hline 124 & 140 & Univ. of Southern Mississippi & -0.227 & 0.870 & 0 & 0.161 & 0.003 & 0 \\
\hline 124 & 173 & Case Western Reserve Univ. & -0.454 & 0.870 & 47 & 0 & 0 & 0 \\
\hline 126 & 98 & Univ. of Pittsburgh & -0.024 & 0.865 & 0 & 0 & 0.052 & 0 \\
\hline 127 & 178 & Boston Univ. & -0.493 & 0.865 & 0 & 0 & 0.009 & 495 \\
\hline 128 & 177 & Clarkson Univ. & -0.490 & 0.864 & 20 & 0 & 0.085 & 4274 \\
\hline 129 & 103 & Catholic Univ. of America & -0.037 & 0.861 & 0 & 0 & 0.055 & 1374 \\
\hline 130 & 165 & Brigham-Young Univ.—Provo & -0.371 & 0.859 & 0 & 0 & 0.029 & 0 \\
\hline 131 & 61 & Univ. of North Carolina_-Greensboro & 0.131 & 0.858 & 0 & 0 & 0 & 0 \\
\hline 132 & 97 & Washington State Univ. & -0.019 & 0.856 & 0 & 0 & 0.028 & 0 \\
\hline 133 & 146 & Univ. of Maryland-Baltimore County & -0.257 & 0.855 & 36 & 0 & 0.001 & 0 \\
\hline 134 & 85 & Oregon State Univ. & 0.043 & 0.854 & 0 & 0 & 0.027 & 0 \\
\hline 135 & 94 & Northeastern Univ. & -0.013 & 0.848 & 0 & 0 & 0 & 1016 \\
\hline 136 & 160 & Univ. of Miami & -0.322 & 0.846 & 0 & 0 & 0.144 & 1578 \\
\hline 137 & 155 & Stevens Institute of Technology & -0.296 & 0.844 & 41 & 0.166 & 0 & 0 \\
\hline 138 & 67 & Univ. of Rhode Island & 0.112 & 0.842 & 0 & 0 & 0 & 0 \\
\hline 139 & 169 & Univ. of Missouri-Columbia & -0.401 & 0.841 & 51 & 0 & 0.167 & 0 \\
\hline 140 & 110 & Ohio State Univ.-Columbus & -0.067 & 0.837 & 0 & 0 & 0.134 & 0 \\
\hline 140 & 176 & Illinois Institute of Technology & -0.487 & 0.837 & 109 & 0.260 & 0 & 0 \\
\hline 142 & 158 & Texas Christian Univ. & -0.305 & 0.836 & 0 & 0 & 0.150 & 3590 \\
\hline 143 & 37 & Andrews Univ. & 0.253 & 0.828 & 0 & 0 & 0.038 & 3624 \\
\hline 144 & 106 & Univ. of Tennessee-Knoxville & -0.047 & 0.825 & 0 & 0 & 0.029 & 0 \\
\hline 145 & 79 & Univ. of Idaho & 0.062 & 0.819 & 0 & 0 & 0 & 0 \\
\hline 145 & 179 & Univ. of California-Santa Cruz & -0.535 & 0.819 & 0 & 0.080 & 0.022 & 0 \\
\hline 147 & 130 & Wichita State Univ. & -0.162 & 0.818 & 0 & 0 & 0 & 77 \\
\hline 147 & 133 & North Carolina State Univ.—Raleigh & -0.167 & 0.818 & 0 & 0 & 0 & 0 \\
\hline 149 & 92 & Univ. of Wyoming & -0.006 & 0.816 & 0 & 0 & 0.021 & 0 \\
\hline 150 & 49 & Temple Univ. & 0.200 & 0.809 & 0 & 0 & 0 & 1093 \\
\hline 150 & 93 & Northern Arizona Univ. & -0.012 & 0.809 & 0 & 0.010 & 0 & 0 \\
\hline 152 & 111 & Univ. of Kansas & -0.079 & 0.807 & 0 & 0 & 0.024 & 0 \\
\hline 153 & 134 & Univ. of Mississippi & -0.169 & 0.804 & 0 & 0.014 & 0.018 & 0 \\
\hline 154 & 142 & Univ. of Kentucky & -0.235 & 0.801 & 0 & 0 & 0.089 & 0 \\
\hline 155 & 89 & Univ. of Utah & 0.025 & 0.799 & 0 & 0 & 0 & 0 \\
\hline 156 & 136 & Univ. of Oklahoma & -0.181 & 0.794 & 0 & 0 & 0 & 0 \\
\hline 157 & 154 & SUNY—Buffalo & -0.291 & 0.792 & 0 & 0 & 0 & 2105 \\
\hline 158 & 162 & Univ. of Alabama-Huntsville & -0.326 & 0.791 & 0 & 0.062 & 0 & 0 \\
\hline 159 & 108 & Old Dominion Univ. & -0.059 & 0.790 & 0 & 0.032 & 0 & 0 \\
\hline 160 & 150 & Drexel Univ. & -0.277 & 0.789 & 0 & 0 & 0 & 1423 \\
\hline 161 & 161 & Montana State Univ. & -0.325 & 0.788 & 0 & 0 & 0.094 & 0 \\
\hline 162 & 122 & Nova Southeastern Univ. & -0.133 & 0.779 & 0 & 0.007 & 0 & 0 \\
\hline 163 & 129 & Texas Tech Univ. & -0.161 & 0.774 & 0 & 0 & 0.041 & 0 \\
\hline 164 & 132 & Florida Institute of Technology & -0.167 & 0.765 & 0 & 0 & 0 & 0 \\
\hline 165 & 182 & Michigan Technological Univ. & -0.577 & 0.762 & 6 & 0 & 0.172 & 0 \\
\hline
\end{tabular}


Appendix 2 continued

\begin{tabular}{|c|c|c|c|c|c|c|c|c|}
\hline \multicolumn{2}{|l|}{ Rank } & \multirow[t]{2}{*}{ School } & \multirow[t]{2}{*}{ Resid. } & \multirow[t]{2}{*}{$\mathrm{TE}$} & \multicolumn{4}{|c|}{ Input slacks } \\
\hline DEA & RES & & & & SAT & Top10 & Full & Cost \\
\hline 166 & 114 & Ball State Univ. & -0.085 & 0.759 & 0 & 0 & 0.071 & 1648 \\
\hline 166 & 159 & SUNY-Stony Brook & -0.307 & 0.759 & 0 & 0 & 0.070 & 1452 \\
\hline 168 & 102 & Virginia Commonwealth Univ. & -0.037 & 0.758 & 0 & 0 & 0 & 0 \\
\hline 169 & 152 & Univ. of Arizona & -0.284 & 0.755 & 0 & 0 & 0.019 & 0 \\
\hline 170 & 139 & Middle Tennessee State Univ. & -0.217 & 0.744 & 0 & 0 & 0 & 0 \\
\hline 171 & 137 & Univ. of South Florida & -0.184 & 0.736 & 0 & 0.065 & 0 & 0 \\
\hline 171 & 148 & Univ. of North Texas & -0.269 & 0.736 & 0 & 0.027 & 0 & 0 \\
\hline 173 & 163 & Univ. of Hawaii_-Manoa & -0.366 & 0.726 & 0 & 0 & 0.073 & 0 \\
\hline 174 & 157 & Arizona State Univ. & -0.303 & 0.719 & 0 & 0.012 & 0 & 0 \\
\hline 175 & 156 & Southern Illinois Univ.-Carbondale & -0.302 & 0.712 & 0 & 0 & 0.012 & 29 \\
\hline 176 & 164 & Univ. of North Dakota & -0.371 & 0.706 & 0 & 0 & 0.111 & 442 \\
\hline 177 & 187 & Univ. of Missouri-Rolla & -1.126 & 0.702 & 40 & 0 & 0.025 & 0 \\
\hline 178 & 175 & Univ. of Arkansas-Fayetteville & -0.475 & 0.701 & 0 & 0.005 & 0 & 0 \\
\hline 179 & 172 & Univ. of Minnesota-Twin Cities & -0.441 & 0.700 & 0 & 0 & 0.062 & 1671 \\
\hline 180 & 170 & Indiana Univ. of Pennsylvania & -0.412 & 0.693 & 0 & 0 & 0.122 & 0 \\
\hline 181 & 128 & Wright State Univ. & -0.157 & 0.681 & 0 & 0 & 0.009 & 0 \\
\hline 182 & 185 & Univ. of Tulsa & -0.860 & 0.679 & 0 & 0 & 0.123 & 510 \\
\hline 183 & 184 & Polytechnic Univ. & -0.675 & 0.676 & 0 & 0.023 & 0 & 5463 \\
\hline 184 & 168 & New Jersey Institute of Technology & -0.398 & 0.667 & 0 & 0 & 0 & 0 \\
\hline 185 & 167 & Univ. of Texas-Arlington & -0.392 & 0.660 & 0 & 0.030 & 0 & 0 \\
\hline 186 & 180 & Univ. of Illinois-Chicago & -0.545 & 0.647 & 0 & 0 & 0.087 & 0 \\
\hline 187 & 174 & Univ. of Alabama-Birmingham & -0.469 & 0.564 & 0 & 0 & 0.007 & 0 \\
\hline
\end{tabular}

